\title{
Association between dietary intake and inflammatory markers: results from the CoLaus study
}

\author{
Eva Piccand ${ }^{1}$, Peter Vollenweider ${ }^{1}$, Idris Guessous ${ }^{2,3}$ and Pedro Marques-Vidal ${ }^{1, *}$ \\ 'Department of Medicine, Internal Medicine, Lausanne University Hospital, Rue du Bugnon 46, 1011 Lausanne, \\ Switzerland: ${ }^{2}$ Department of Ambulatory Care and Community Medicine, University of Lausanne, Lausanne, \\ Switzerland: ${ }^{3}$ Department of Community Medicine, Preventive Care and Emergency Medicine, Geneva University \\ Hospitals, Geneva, Switzerland
}

Submitted 4 April 2018: Final revision received 18 July 2018: Accepted 7 August 2018: First published online 18 October 2018

\begin{abstract}
Objective: To assess the associations between single foods, nutrients, dietary patterns and dietary scores, and inflammatory markers (C-reactive protein (CRP), IL-6, TNF- $\alpha$ and leucocyte count).

Design: Cross-sectional, population-based study.

Setting: City of Lausanne, Switzerland, years 2009-2012.

Subjects: Adults ( $n$ 4027; 46.5\% men), mean age $57 \cdot 2$ (sD 10.2) years. Dietary intake was collected using a semi-quantitative FFQ. Single foods and nutrients, three dietary patterns ('Meat \& fries'; 'Fruits \& vegetables'; 'Fatty \& sugary') and three dietary scores (two Mediterranean; Alternative Healthy Eating Index (AHEI)) were used. Associations were assessed using correlation and multivariable linear regression.

Results: After adjusting for total energy intake, gender and other sociodemographic factors, no individual macro- or micronutrient was associated with inflammatory markers. Among single foods, only fruit intake was negatively associated with CRP levels (standardized regression score $=-0.043, P<0 \cdot 01$ ). The 'Fruits \& vegetables' pattern, the Mediterranean and the AHEI scores were negatively associated with CRP levels (standardized regression score $=-0.079,-0.043$ and -0.067 , respectively, all $P<0 \cdot 01$ ). When entered simultaneously with fruit intake, the 'Fruits \& vegetables' pattern, the Mediterranean and the AHEI scores tended to remain significantly and negatively associated with CRP levels, while the association with fruit intake was no longer significant. No association between all dietary markers and IL-6, TNF- $\alpha$ or leucocyte count was found.

Conclusions: Dietary scores, but not individual foods, are associated with inflammatory markers in the general population.
\end{abstract}

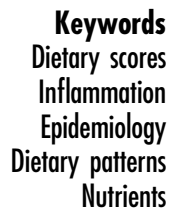

Inflammatory markers such as C-reactive protein (CRP) and IL- 6 are predictors of CVD risk ${ }^{(1)}$. Indeed, the wellknown association between diet and CVD may partly be linked through these inflammatory markers ${ }^{(2)}$. Several epidemiological studies have identified negative associations between inflammatory levels and specific nutrients such as polyphenols ${ }^{(3,4)}$, PUFA $^{(5)}$, histidine ${ }^{(6)}$ and branched-chain amino acids ${ }^{(7)}$.

Besides specific nutrients, dietary scores based on the consumption of specific food items have also been linked with inflammatory levels. For example, hypothesisoriented scores such as the Mediterranean diet score ${ }^{(8)}$ have been linked with lower platelet and white blood cell counts ${ }^{(9)}$. Similarly, the Southern European Atlantic Diet $^{(10)}$ and the Baltic Sea diet ${ }^{(11)}$ have been associated with lower levels of CRP, while the Alternative Healthy Eating Index (AHEI) of McCullough et al. ${ }^{(12)}$ was negatively associated with IL-6 levels ${ }^{(2,13)}$.

Dietary patterns obtained through principal component analysis have also been shown to be associated with inflammatory levels. For example, 'prudent'(14) or 'healthaware $^{(15)}$ patterns were negatively associated with CRP levels. Ozawa et al. used reduced rank regression analysis and identified a 'dietary inflammatory pattern' rich in red and processed meat, peas, legumes and fried food, and low in whole grains, which was positively associated with IL-6 levels and other inflammatory markers ${ }^{(16)}$. However, most studies assessed only a limited number of nutrients or foods, or even focused on a single dietary score or pattern, while studies comparing simultaneously the 
associations between different dietary factors and inflammatory markers are scarce ${ }^{(9,15,17,18)}$.

Hence, the present study aimed at assessing the associations between a wide range of dietary factors (macroand micronutrients, single foods, dietary patterns and scores) and several inflammatory markers. Our hypothesis was that dietary patterns would be more associated with inflammatory markers than individual foods or nutrients.

\section{Participants and methods}

\section{Participants}

The CoLaus study is a population-based study assessing the clinical, biological and genetic determinants of CVD in the city of Lausanne, Switzerland ${ }^{(19)}$. The sampling procedure of the CoLaus study was as follows: the source population was defined as all individuals aged between 35 and 75 years registered in the population register of the city of Lausanne. The register includes all individuals living in this city for more than $90 \mathrm{~d}$. A simple, non-stratified random sample of 19830 individuals (corresponding to $35 \%$ of the source population) was drawn and the selected individuals were invited to participate by letter. If no answer was obtained, a second letter was sent and if no answer was obtained, the individuals were contacted by telephone. The following inclusion criteria were applied: (i) written informed consent; and (ii) willingness to take part in the examination and to provide blood samples.

Recruitment of the baseline sample began in June 2003 and ended in May 2006, enrolling 6733 participants who underwent an interview, a physical examination and a blood analysis. The first follow-up was performed between April 2009 and September 2012, 5.6 years on average after collection of the baseline data, and included 5064 of the initial participants. The information collected in the first follow-up was similar to that collected in the baseline examination except that in the first follow-up information regarding food consumption and detailed physical activity was also collected. Hence, for the present study, only data from the first follow-up examination were considered, as dietary intake assessment was first introduced then.

\section{Blood samples}

Venous blood samples $(50 \mathrm{ml})$ were drawn in the fasting state. High-sensitive CRP (hs-CRP) was assessed by immunoassay and latex HS (IMMULITE 1000-High; Diagnostic Products Corporation, Los Angeles, CA, USA) with maximum intra- and inter-batch $\mathrm{CV}$ of 1.3 and $4.6 \%$, respectively. Serum samples were kept at $-80^{\circ} \mathrm{C}$ before assessment of IL- 6 and TNF- $\alpha$ and sent on dry ice to the laboratory. Levels of these cytokines were measured using a multiplexed particle-based flow cytometric cytokine assay ${ }^{(20)}$. Milliplex kits were purchased from Millipore
(Zug, Switzerland). The procedures closely followed the manufacturer's instructions. The analysis was conducted using a conventional flow cytometer (FC500 MPL; Beckman Coulter, Nyon, Switzerland). Lower limits of detection for IL- 6 and TNF- $\alpha$ were $0.2 \mathrm{pg} / \mathrm{ml}$. A good agreement between signal and cytokine was found within the assay range $\left(R^{2} \geq 0 \cdot 99\right)$. Intra- and inter-assay $\mathrm{CV}$ were respectively 16.9 and $16.1 \%$ for IL- 6 and 12.5 and $13.5 \%$ for TNF- $\alpha$. For quality control, repeated measurements were conducted in eighty participants randomly drawn from the initial sample. Spearman rank correlations between duplicate measurements were 0.961 and 0.891 for IL- 6 and TNF- $\alpha$, respectively (all $P<0.001$ ). Lin's correlation coefficients were 0.971 and 0.945 and intra-class correlation coefficients were 0.972 and 0.946 for IL- 6 and TNF- $\alpha$, respectively (all $P<0 \cdot 001$ ), indicating a good reproducibility.

\section{Dietary intake}

Dietary intake was assessed using a validated self-administered, semi quantitative FFQ which also included portion size ${ }^{(21)}$. This FFQ has been validated against $24 \mathrm{~h}$ recalls among 626 volunteers from the Geneva population $^{(21-23)}$. The FFQ assesses the dietary intake of the previous 4 weeks and consists of ninety-seven different food items accounting for more than $90 \%$ of the intake of energy, proteins, fat, carbohydrates, alcohol, cholesterol, vitamin D and retinol, and $85 \%$ of fibre, carotene and Fe. For each item, seven consumption frequencies were provided: (i) 'less than once during the last 4 weeks'; (ii) 'once per month'; (iii) ' $2-3$ times per month'; (iv) ' $1-2$ times per week'; (v) '3-4 times per week'; (vi) 'once per day'; and (vii) ' 2 or more times per day'. Participants indicated the average serving size (smaller, equal or larger) compared with a reference size. Daily consumption of the different food items was computed based on frequency and portion size and expressed in millilitres (for drinks) or grams (for other foods). Although the maximum frequency was ' 2 or more times per day', there were six items assessing fruit intake and eight items assessing vegetable intake. Hence, in the unlikely case a participant would eat fruits or vegetables pertaining to a single item (for example, only berries), the multiplicity of the items related to fruits and vegetables would allow an adequate estimation of their intake. Conversion into nutrients was performed based on the French CIQUAL food composition table ${ }^{(24)}$ taking account of portion size.

Three hypothesis-oriented dietary scores were computed, two based on the Mediterranean diet, the third on a modification of the AHEI. The first Mediterranean dietary score (hereby designated as 'Mediterranean score 1') was derived from Trichopoulou et al. ${ }^{(8)}$; the score ranges between 0 and 8 . The second Mediterranean dietary score (hereby designated as 'Mediterranean score 2') is adapted to the Swiss population and 
was computed according to Vormund et al. ${ }^{(25)}$. Contrary to the score from Trichopoulou et al., dairy products are considered as beneficial. The score thus ranges between 0 and 9. The AHEI was adapted from McCullough et al. ${ }^{(12)}$. In our study, the amount of trans fat could not be assessed, and we considered all participants taking multivitamins as taking them for a duration $\geq 5$ years. Thus, the modified AHEI score ranged between 2.5 and 77.5 , instead of 2.5 and 87.5 for the original AHEI score ${ }^{(12)}$. For all three scores, higher values represented a healthier diet.

A restricted version of the Dietary Inflammatory Index (DII) was computed using available data and the corresponding overall inflammatory effect scores ${ }^{(26)}$. The higher the index, the higher its inflammatory capacity. The procedures to compute the three hypothesis-oriented dietary scores and the DII are summarized in the online supplementary material, Supplemental Table 1.

Dietary patterns were derived using principal component analysis based on food consumption frequencies. Three dietary patterns were identified: 'Meat \& fries', 'Fruits \& vegetables' and 'Fatty \& sugary'. Detailed description of assessment and characteristics of the dietary patterns is provided elsewhere ${ }^{(27)}$.

\section{Other covariates}

Sociodemographic and lifestyle data were collected by self-administered questionnaires. Educational level was categorized as low (primary), middle (apprenticeship or secondary school) and high (university). Smoking status was categorized as never, former (irrespective of the time since quitting) and current (irrespective of the amount smoked). Physical activity was assessed by a questionnaire validated in the population of Geneva ${ }^{(28)}$. This selfreported questionnaire assesses the type and duration of seventy kinds of (non-)professional activities and sports during the previous week. Sedentary status was defined as spending more than $90 \%$ of the daily energy in activities below moderate and high intensity (defined as requiring at least $4 \times \mathrm{BMR}^{(29)}$ and categorized as a dichotomous variable (yes/no). BMR multiples are close to metabolic equivalent of task (MET) multiples, although MET multiples do not take participant sex, age or height into account.

Body weight and height were measured with participants standing without shoes in light indoor clothing. Weight was measured in kilograms to the nearest $0 \cdot 1 \mathrm{~kg}$ using a Seca ${ }^{\mathrm{TM}}$ scale and height was measured to the nearest $5 \mathrm{~mm}$ using a Seca ${ }^{\mathrm{TM}}$ height gauge (Seca, Hamburg, Germany). BMI was defined as weight $/$ height $^{2}$ and categorized as normal (BMI $<25 \cdot 0 \mathrm{~kg} / \mathrm{m}^{2}$ ); overweight $\left(25 \cdot 0 \leq \mathrm{BMI}<30 \cdot 0 \mathrm{~kg} / \mathrm{m}^{2}\right)$ and obese (BMI $\geq 30 \cdot 0$ $\mathrm{kg} / \mathrm{m}^{2}$ ). Due to small numbers ( $n$ 72), underweight participants $\left(\mathrm{BMI}<18.5 \mathrm{~kg} / \mathrm{m}^{2}\right)$ were included in the 'normal' category.

\section{Exclusion criteria}

Participants with the following characteristics were excluded: (i) no dietary data; (ii) no socio-clinical data; (iii) no inflammatory data; (iv) anti-inflammatory drugs; (v) inflammation (CRP $>20 \mathrm{mg} / \mathrm{l}$ ); and (vi) total energy intake of $<3556$ or $>18828 \mathrm{~kJ} / \mathrm{d}(<850$ or $>4500 \mathrm{kcal} / \mathrm{d})$.

\section{Statistical analysis}

Statistical analysis was performed using the statistical software package Stata version 15.1 for Windows. Participants' characteristics were expressed as number and percentage for categorical variables, or as mean and standard deviation for continuous variables.

Bivariate associations were assessed using Spearman non-parametric rank correlation. Dietary markers significantly associated on bivariate analysis with inflammatory markers were further explored using multivariable analysis. Multivariable analysis was performed using linear regression adjusting for age (continuous), BMI (continuous), gender, smoking (never, former, current), educational level (high, middle, low), sedentary status (yes/no), diabetes (yes/no) and total energy intake (continuous). Results were expressed as standardized linear regression coefficients, which can be interpreted as multivariable-adjusted correlations. For multivariable analyses, inflammatory markers were log-transformed.

The importance of dietary scores and patterns relative to single foods was further addressed by entering simultaneously in each model one dietary pattern and the foods significantly associated with inflammatory markers. Given the number of associations tested, statistical significance was considered for a two-sided test with $P<0.01$ to reduce the false positive detection rate.

\section{Results}

\section{Characteristics of the participants}

Of the initial 5064 participants, 1037 (20.5\%) were excluded. The reasons for exclusion are indicated in Fig. 1 and the characteristics of excluded and included participants are summarized in the online supplementary material, Supplemental Table 2. Excluded participants were older, had a higher BMI, a lower education, and were prone to smoke, be sedentary and have diabetes.

\section{Associations of individual nutrients with inflammatory markers}

On bivariate analysis, PUFA was positively associated with IL-6, and carotene was negatively associated with CRP and leucocyte count (Table 1). However, these relationships were no longer significant when adjusting for confounders (Table 2). 


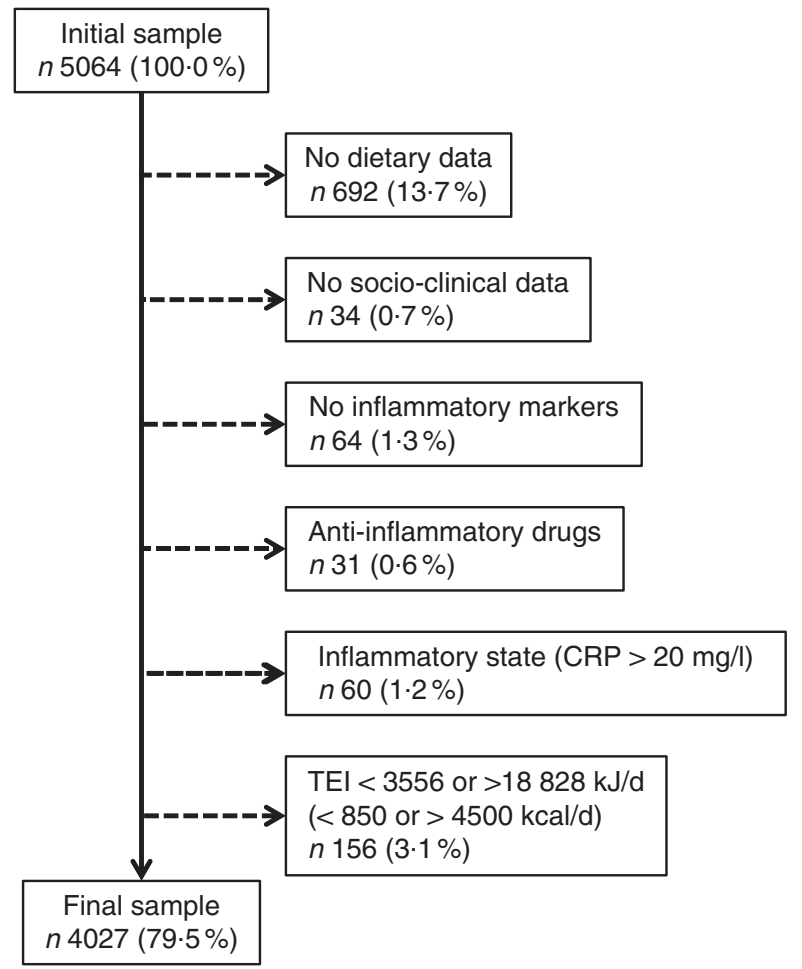

Fig. 1 Selection of participants for the present study (CRP, C-reactive protein; TEI, total energy intake)

\section{Associations of single foods with inflammatory markers}

On bivariate analysis, fruits, carrots and tofu were negatively associated with CRP and leucocyte count; green salad, bananas, apples and kiwis were negatively associated with leucocyte count, while berries showed a negative association with IL-6 levels (Table 1). After multivariate adjustment, only the negative association between fruits and CRP retained statistical significance (Table 2).

\section{Associations of dietary patterns and scores with inflammatory markers}

On bivariate analysis, the 'Meat \& fries' pattern was positively associated and the 'Fruits \& vegetables' pattern was negatively associated with CRP levels and leucocyte count (Table 1). After multivariable adjustment, only the negative association between the 'Fruits \& vegetables' pattern and CRP levels remained (Table 2).

On bivariate analysis, both Mediterranean scores and the AHEI were negatively associated with CRP levels; the AHEI was also negatively associated with leucocyte count (Table 1). After multivariable adjustment, only the negative associations of Mediterranean score 1 and the AHEI with CRP levels remained (Table 2). The DII was positively associated with CRP levels and leucocyte count on bivariate analysis (Table 1), but those associations were no longer significant after multivariable adjustment (Table 2).
When entered simultaneously with fruit intake, the 'Fruits \& vegetables' pattern, Mediterranean score 1 and the AHEI tended to remain significantly and negatively associated with CRP levels, while the association with fruit intake was no longer significant (see online supplementary material, Supplemental Table 3).

\section{Discussion}

The present study is one of the few that compared the associations between different dietary factors and inflammatory markers in the general population. Our results show that (un)healthy dietary behaviours are associated with inflammatory markers, while individual nutrients or foods are not.

\section{Associations of individual nutrients with inflammatory markers}

Only a limited number of macro- and micronutrients were associated with inflammatory markers on bivariate analysis, and no significant association remained after adjustment for confounders. These findings are in agreement with a cross-sectional Scottish study ${ }^{(15)}$, which also failed to find any significant association between several micronutrients (flavonoids and antioxidants) and inflammatory markers. Conversely, they do not replicate a literature review where large numbers of foods and nutrients were found to be associated (either positively or negatively) with inflammatory markers ${ }^{(26)}$. It would be important to assess which of all these foods and nutrients are significantly and independently associated with inflammatory markers, via multivariable analyses.

\section{Associations of single foods with inflammatory markers}

Fruit intake was negatively associated with CRP levels, but not with IL-6, TNF- $\alpha$ or leucocyte count. The association with CRP remained after multivariable analysis, a finding in agreement with two cross-sectional studies conducted in Portugal ${ }^{(18)}$ and Iran $^{(30)}$. A possible explanation is the high polyphenol content of fruits ${ }^{(31)}$, which has been linked with a decrease in inflammation levels ${ }^{(3,4)}$. Our results thus suggest that an adequate consumption of fruit could decrease inflammatory levels.

\section{Associations of dietary patterns and scores with inflammatory markers}

The Mediterranean diet scores were negatively associated with CRP levels, and this association persisted for Mediterranean score 1 after multivariable adjustment. These findings agree with previous cross-sectional studies ${ }^{(9,32)}$, suggesting that the beneficial effect of the Mediterranean 
Table 1 Bivariate associations between inflammatory and dietary markers; CoLaus study, Lausanne, Switzerland, 2009-2012

\begin{tabular}{|c|c|c|c|c|}
\hline & CRP & IL-6 & TNF-a & Leucocytes \\
\hline Total energy intake & -0.003 & 0.046 & 0.018 & 0.010 \\
\hline \multicolumn{5}{|l|}{ Macronutrients } \\
\hline Total protein & -0.002 & 0.039 & 0.024 & 0.025 \\
\hline Vegetable protein & -0.040 & 0.030 & -0.003 & -0.031 \\
\hline Animal protein & 0.018 & 0.031 & 0.033 & 0.049 \\
\hline Total carbohydrates & -0.031 & 0.023 & 0.008 & -0.010 \\
\hline Monosaccharides & -0.036 & 0.007 & -0.001 & -0.015 \\
\hline Polysaccharides & -0.018 & 0.029 & 0.012 & -0.008 \\
\hline Total fat & 0.002 & 0.044 & 0.019 & 0.017 \\
\hline SFA & 0.009 & 0.035 & 0.035 & 0.024 \\
\hline MUFA & -0.007 & 0.045 & 0.011 & 0.014 \\
\hline PUFA & 0.034 & $0.057^{\star}$ & 0.011 & 0.041 \\
\hline Fibre & -0.049 & 0.015 & -0.006 & $-0.055^{\star}$ \\
\hline Cholesterol & -0.007 & 0.019 & 0.003 & 0.010 \\
\hline Alcohol & -0.022 & 0.041 & 0.026 & 0.010 \\
\hline \multicolumn{5}{|l|}{ Micronutrients } \\
\hline $\mathrm{Ca}$ & -0.016 & 0.016 & 0.022 & -0.029 \\
\hline $\mathrm{Fe}$ & -0.020 & 0.043 & 0.008 & -0.013 \\
\hline Retinol & 0.016 & 0.017 & 0.011 & 0.014 \\
\hline Carotene & $-0.059^{\star}$ & 0.021 & -0.012 & $-0.082^{*}$ \\
\hline Vitamin D & -0.027 & 0.020 & -0.002 & -0.026 \\
\hline Vitamin A & -0.018 & 0.028 & -0.005 & -0.027 \\
\hline \multicolumn{5}{|l|}{ Food items } \\
\hline Fruits & $-0.057^{*}$ & -0.009 & -0.026 & $-0.065^{\star}$ \\
\hline Vegetables & -0.044 & 0.049 & -0.007 & -0.040 \\
\hline Fish & -0.040 & 0.019 & -0.004 & -0.020 \\
\hline Green beans & -0.016 & 0.004 & 0.000 & 0.008 \\
\hline Cauliflower & -0.026 & 0.032 & -0.006 & -0.022 \\
\hline Tomatoes & 0.014 & -0.005 & 0.005 & 0.009 \\
\hline Carrots & $-0.057^{*}$ & 0.017 & 0.000 & $-0.096^{\star}$ \\
\hline Green salad & -0.043 & 0.017 & -0.020 & $-0.058^{\star}$ \\
\hline Thick vegetable soup & -0.031 & 0.032 & -0.013 & 0.001 \\
\hline Tomato sauce & -0.026 & 0.004 & -0.032 & 0.018 \\
\hline Tofu & $-0.089^{\star}$ & 0.015 & -0.022 & $-0.082^{\star}$ \\
\hline Bananas, apples & -0.036 & -0.014 & 0.004 & $-0.054^{\star}$ \\
\hline Citrus fruits & -0.020 & -0.002 & -0.030 & -0.022 \\
\hline Peaches, nectarines & 0.027 & -0.048 & 0.044 & 0.021 \\
\hline Berries & 0.024 & $-0.055^{\star}$ & -0.012 & -0.022 \\
\hline Kiwis & -0.031 & 0.013 & 0.015 & $-0.079^{\star}$ \\
\hline Fresh fruit juice & -0.045 & -0.035 & -0.037 & -0.011 \\
\hline \multicolumn{5}{|l|}{ Patterns } \\
\hline 'Meat \& fries' & $0.073^{*}$ & 0.025 & 0.010 & $0.110^{\star}$ \\
\hline 'Fruits \& vegetables' & $-0.095^{\star}$ & 0.021 & -0.036 & $-0.104^{*}$ \\
\hline 'Fatty \& sugary' & 0.016 & 0.002 & 0.005 & 0.015 \\
\hline \multicolumn{5}{|l|}{ Dietary scores } \\
\hline Mediterranean score $1 \dagger$ & $-0.076^{\star}$ & 0.036 & -0.025 & -0.039 \\
\hline Mediterranean score 2‡ & $-0.058^{\star}$ & 0.029 & -0.016 & -0.043 \\
\hline $\mathrm{AHEI}$ & $-0 \cdot 110^{\star}$ & 0.026 & -0.020 & $-0.075^{\star}$ \\
\hline DII & $0.055^{\star}$ & -0.018 & 0.015 & $0.081^{*}$ \\
\hline
\end{tabular}

CRP, C-reactive protein; AHEI, Alternative Healthy Eating Index; DII, Dietary Inflammatory Index.

Data from 4027 participants. Results are expressed as Spearman correlations.

* Significant correlation: $P<0.01$.

†Mediterranean diet score of Trichopoulou et al. ${ }^{(8)}$.

¥Mediterranean diet score of Vormund et al. ${ }^{(25)}$.

diet on CVD might partly be linked to a decreased inflammatory status.

The AHEI was negatively associated with CRP levels. This finding concurs with some prospective and crosssectional studies where the AHEI was inversely associated with CRP $^{(33)}$ or IL-6 levels ${ }^{(2,13)}$, but not with other crosssectional or case-control studies which failed to find any association $^{(34,35)}$. A possible explanation for the lack of association in the latter studies is their relatively small sample size (<1000 participants), which might have reduced statistical power.
Of the three dietary patterns obtained, only the 'Fruits \& vegetables' retained its negative association with CRP after multivariable adjustment. These findings are partly in agreement with other studies, which also assessed dietary patterns using principal component analysis. Indeed, both the 'health-aware' pattern from the Lothian Birth Cohort cross-sectional study ${ }^{(15)}$ and the 'prudent' pattern from the Aberdeen Prospective Osteoporosis Screening Study cohort ${ }^{(14)}$, which scored high in fruits and vegetables, were negatively associated with CRP levels. 
Table 2 Multivariable associations between selected inflammatory and dietary markers; CoLaus study, Lausanne, Switzerland, 20092012

\begin{tabular}{|c|c|c|c|}
\hline & CRP (log) & IL-6 (log) & Leucocytes \\
\hline \multicolumn{4}{|l|}{ Macronutrients } \\
\hline Total fat & - & - & - \\
\hline MUFA & - & - & - \\
\hline PUFA & - & 0.005 & - \\
\hline Fibre & - & - & -0.028 \\
\hline \multicolumn{4}{|l|}{ Micronutrients } \\
\hline Carotene & -0.037 & - & -0.047 \\
\hline \multicolumn{4}{|l|}{ Food items } \\
\hline Fruits & $-0.043^{*}$ & - & -0.026 \\
\hline Vegetables & - & - & - \\
\hline Carrots & -0.037 & - & -0.041 \\
\hline Green salad & - & - & -0.043 \\
\hline Tofu & -0.024 & - & -0.013 \\
\hline Bananas, apples & - & - & -0.010 \\
\hline Berries & - & -0.004 & - \\
\hline Kiwis & - & - & -0.016 \\
\hline \multicolumn{4}{|l|}{ Dietary patterns } \\
\hline 'Meat \& fries' & 0.033 & - & 0.035 \\
\hline 'Fruits \& vegetables' & $-0.079^{\star}$ & - & -0.044 \\
\hline 'Fatty \& sugary' & - & - & - \\
\hline \multicolumn{4}{|l|}{ Dietary scores } \\
\hline Mediterranean score $1 \dagger$ & $-0.043^{*}$ & - & - \\
\hline Mediterranean score $2 \ddagger$ & -0.039 & - & - \\
\hline AHEI & $-0.067^{\star}$ & - & -0.041 \\
\hline DII & 0.036 & - & 0.047 \\
\hline
\end{tabular}

CRP, C-reactive protein; AHEI, Alternative Healthy Eating Index; DII, Dietary Inflammatory Index; -, not assessed.

Data from 4027 participants. Results are expressed as standardized regression coefficients adjusted for age (continuous), BMI (continuous), gender, smoking (never, former, current), educational level (high, middle, low), sedentary status (yes/no), diabetes (yes/no) and total energy intake (continuous)

*Significant association: $P<0.01$

†Mediterranean diet score of Trichopoulou et al. ${ }^{(8)}$.

$\ddagger$ Mediterranean diet score of Vormund et al. ${ }^{(25)}$.

Interestingly, the 'Fruits \& vegetables' pattern, Mediterranean score 1 and the AHEI showed stronger negative associations with CRP levels than single fruit intake, indicating that the associations were due to not only a higher fruit intake, but also that other food items related to the pattern/score might intervene. Overall, our results suggest that a diet rich in fruits (but not only) is associated with lower inflammatory levels.

No associations were found between the DII and inflammatory markers after multivariable adjustment. A likely explanation is that the DII computed in the present study was based on only fourteen of the forty-five items that constitute the original DII (see online supplementary material, Supplemental Table 1) ${ }^{(26)}$. This might have led to inadequate DII values and thus biased estimates. Indeed, most items not included in the computation had a negative score (Supplemental Table 1). Future studies will have to assess the validity of DII computed using a limited data set.

\section{Importance for clinical practice}

Many studies have focused on the associations between single nutrients or foods and inflammatory markers. Still, increasing or decreasing the consumption of specific nutrients or of selected foods might be difficult to achieve in general practice. Our results indicate that dietary recommendations focused on the consumption of several food groups are more important than recommendations focused on specific foods or nutrients ${ }^{(36)}$. Hence, in clinical practice, generic recommendations could be provided, instead of focusing on specific foods or nutrients, which are difficult to identify and to integrate in a normal diet. This would facilitate dietary counselling by general practitioners, whose nutritional knowledge is usually low ${ }^{(37)}$.

Similarly, from a public health perspective, simple messages aimed at a healthier eating and increased consumption of fruits and vegetables ${ }^{(38)}$ could be delivered. The impact of such measures in the general population could then be monitored by any of the scores (AHEI, Mediterranean or 'Fruits \& vegetables') rather than by complex nutrient assessment.

\section{Strengths and limitations}

Our study has several strengths. First, it is one of the very few simultaneously comparing the associations between different dietary factors and inflammatory markers ${ }^{(15)}$. Second, due to the population-based setting, our results can be transposed to other populations and practical recommendations can be used in public health and clinical practice.

The study also has several limitations. First, and as already indicated, no information regarding polyphenols was available. Hence, it was not possible to confirm previous findings ${ }^{(3,4)}$. Future studies should rely either on an extensive food composition database or on the direct measurement of polyphenols in serum or urine. Second, Mediterranean score 1 is based on a Greek population's food consumption, and the scores obtained cannot be directly transposed to a Swiss population. Third, the crosssectional setting of the study does not allow establishing a temporal relationship between dietary intake and inflammatory markers. Still, they are in line with the results of randomized controlled trials showing that an increased consumption of flavonoid-rich fruits and vegetables ${ }^{(39)}$ or whole-grain wheat ${ }^{(40)}$ decreases inflammatory markers. Finally, the FFQ assessed dietary intake for the last 4 weeks and not for the whole year. Still, it has been shown that FFQ assessing short periods do not differ from FFQ assessing long periods ${ }^{(41)}$, while minimizing recall bias.

\section{Conclusion}

Our results show that healthy dietary behaviours, but not individual foods, are negatively associated with inflammatory markers in the general population. 


\section{Acknowledgements}

Financial support: The CoLaus study was and is supported by research grants from GlaxoSmithKline, the Faculty of Biology and Medicine of Lausanne, and the Swiss National Science Foundation (grants numbers 33CSCO-122661, 33CS30-139468 and 33CS30-148401). The funding sources had no involvement in the study design, conduct of the study, data collection, analysis of samples or data, interpretation of findings, writing of the report, or decision to submit the article for publication. Conflict of interest: The authors report no conflict of interest. Authorship: E.P. performed part of the statistical analyses and wrote most of the article; P.M.-V. collected data, performed most of the statistical analysis and wrote part of the article; P.V. and I.G. revised the article for important intellectual content. P.M.-V. had full access to the data and is the guarantor of the study. Ethics of buman subject participation: The institutional Ethics Committee of the University of Lausanne, which afterwards became the Ethics Commission of Canton Vaud (www.cer-vd.ch), approved the baseline CoLaus study (reference 16/03, decisions of 13 January and 10 February 2003); the approval was renewed for the first (reference 33/09, decision of 23 February 2009) and the second (reference 26/14, decision of 11 March 2014) follow-ups. The full decisions of the CER-VD can be obtained from the authors upon request. The study was performed in agreement with the Declaration of Helsinki and its former amendments, and in accordance with the applicable Swiss legislation. All participants gave their signed informed consent before entering the study.

\section{Supplementary material}

To view supplementary material for this article, please visit https://doi.org/10.1017/S1368980018002355

\section{References}

1. Luc G, Bard J-M, Juhan-Vague I et al. (2003) C-reactive protein, interleukin- 6 , and fibrinogen as predictors of coronary heart disease: the PRIME study. Arterioscler Thromb Vasc Biol 23, 1255-1261.

2. Fung TT, McCullough ML, Newby PK et al. (2005) Dietquality scores and plasma concentrations of markers of inflammation and endothelial dysfunction. Am J Clin Nutr 82, 163-173.

3. Cassidy A, Rogers G, Peterson JJ et al. (2015) Higher dietary anthocyanin and flavonol intakes are associated with antiinflammatory effects in a population of US adults. Am J Clin Nutr 102, 172-181.

4. Pounis G, Bonaccio M, Di Castelnuovo A et al. (2016) Polyphenol intake is associated with low-grade inflammation, using a novel data analysis from the Moli-sani study. Thromb Haemost 115, 344-352.

5. Muka T, Kiefte-de Jong JC, Hofman A et al. (2015) Polyunsaturated fatty acids and serum C-reactive protein: the Rotterdam study. Am J Epidemiol 181, 846-856.
6. Li YC, Li CL, Qi JY et al. (2016) Relationships of dietary histidine and obesity in northern Chinese adults, an internetbased cross-sectional study. Nutrients $\mathbf{8}, 420$.

7. Li YC, Li Y, Liu LY et al. (2015) The ratio of dietary branched-chain amino acids is associated with a lower prevalence of obesity in young northern Chinese adults: an internet-based cross-sectional study. Nutrients $\mathbf{7}$, 9573-9589.

8. Trichopoulou A, Costacou T, Bamia C et al. (2003) Adherence to a Mediterranean diet and survival in a Greek population. New Engl J Med 348, 2599-2608.

9. Bonaccio M, Di Castelnuovo A, De Curtis A et al. (2014) Adherence to the Mediterranean diet is associated with lower platelet and leukocyte counts: results from the Molisani study. Blood 123, 3037-3044.

10. Guallar-Castillón P, Oliveira A, Lopes C et al. (2013) The Southern European Atlantic Diet is associated with lower concentrations of markers of coronary risk. Atherosclerosis 226, 502-509.

11. Kanerva N, Kaartinen NE, Rissanen H et al. (2014) Associations of the Baltic Sea diet with cardiometabolic risk factors - a meta-analysis of three Finnish studies. Br J Nutr 112, 616-626.

12. McCullough ML, Feskanich D, Stampfer MJ et al. (2002) Diet quality and major chronic disease risk in men and women: moving toward improved dietary guidance. Am J Clin Nutr 76, 1261-1271.

13. Akbaraly TN, Shipley MJ, Ferrie JE et al. (2015) Long-term adherence to healthy dietary guidelines and chronic inflammation in the prospective Whitehall II study. Am J Med 128, 152-160.

14. Wood AD, Strachan AA, Thies F et al. (2014) Patterns of dietary intake and serum carotenoid and tocopherol status are associated with biomarkers of chronic low-grade systemic inflammation and cardiovascular risk. Br J Nutr 112, 1341-1352.

15. Corley J, Kyle JAM, Starr JM et al. (2015) Dietary factors and biomarkers of systemic inflammation in older people: the Lothian Birth Cohort 1936. Br J Nutr 114, 10881098.

16. Ozawa M, Shipley M, Kivimaki M et al. (2017) Dietary pattern, inflammation and cognitive decline: the Whitehall II prospective cohort study. Clin Nutr 36, 506-512.

17. Nettleton JA, Matijevic N, Follis JL et al. (2010) Associations between dietary patterns and flow cytometry-measured biomarkers of inflammation and cellular activation in the Atherosclerosis Risk in Communities (ARIC) Carotid Artery MRI Study. Atherosclerosis 212, 260-267.

18. Oliveira A, Rodriguez-Artalejo F \& Lopes C (2009) The association of fruits, vegetables, antioxidant vitamins and fibre intake with high-sensitivity C-reactive protein: sex and body mass index interactions. Eur J Clin Nutr 63 , 1345-1352.

19. Firmann M, Mayor V, Vidal PM et al. (2008) The CoLaus study: a population-based study to investigate the epidemiology and genetic determinants of cardiovascular risk factors and metabolic syndrome. BMC Cardiovasc Disord $8,6$.

20. Vignali DA (2000) Multiplexed particle-based flow cytometric assays. J Immunol Methods 243, 243-255.

21. Bernstein L, Huot I \& Morabia A (1995) Amélioration des performances d'un questionnaire alimentaire semiquantitatif comparé à un rappel des 24 heures. Sante Publique 7, 403-413.

22. Morabia A, Bernstein M, Heritier S et al. (1997) Communitybased surveillance of cardiovascular risk factors in Geneva: methods, resulting distributions, and comparisons with other populations. Prev Med 26, 311-319.

23. Beer-Borst $\mathrm{S}$, Costanza MC, Pechère-Bertschi A et al. (2009) Twelve-year trends and correlates of dietary salt intakes for 
the general adult population of Geneva, Switzerland. Eur J Clin Nutr 63, 155-164.

24. French Agency for Food, Environmental and Occupational Health \& Safety (2016) ANSES-CIQUAL French food composition table. https://ciqual.anses.fr/ (accessed August 2018).

25. Vormund K, Braun J, Rohrmann S et al. (2015) Mediterranean diet and mortality in Switzerland: an alpine paradox? Eur J Nutr 54, 139-148.

26. Shivappa N, Steck SE, Hurley TG et al. (2014) Designing and developing a literature-derived, population-based dietary inflammatory index. Public Health Nutr 17, 1689-1696.

27. Marques-Vidal P, Waeber G, Vollenweider P et al. (2018) Socio-demographic and lifestyle determinants of dietary patterns in French-speaking Switzerland, 2009-2012. BMC Public Health 18, 131.

28. Bernstein M, Sloutskis D, Kumanyika S et al. (1998) Databased approach for developing a physical activity frequency questionnaire. Am J Epidemiol 147, 147-154.

29. Guessous I, Gaspoz JM, Theler JM et al. (2014) Eleven-year physical activity trends in a Swiss urban area. Prev Med 59, 25-30.

30. Esmaillzadeh A, Kimiagar M, Mehrabi Y et al. (2006) Fruit and vegetable intakes, C-reactive protein, and the metabolic syndrome. Am J Clin Nutr 84, 1489-1497.

31. Pérez-Jiménez J, Neveu V, Vos F et al. (2010) Identification of the 100 richest dietary sources of polyphenols: an application of the Phenol-Explorer database. Eur J Clin Nutr 64, Suppl. 3, S112-S120.

32. Chrysohoou C, Panagiotakos DB, Pitsavos C et al. (2004) Adherence to the Mediterranean diet attenuates inflammation and coagulation process in healthy adults: the ATTICA study. J Am Coll Cardiol 44, 152-158.

33. Fargnoli JL, Fung TT, Olenczuk DM et al. (2008) Adherence to healthy eating patterns is associated with higher circulating total and high-molecular-weight adiponectin and lower resistin concentrations in women from the Nurses' Health Study. Am J Clin Nutr 88, 1213-1224.

34. Huang T, Tobias DK, Hruby A et al. (2016) An increase in dietary quality is associated with favorable plasma biomarkers of the brain-adipose axis in apparently healthy US women. J Nutr 146, 1101-1108.

35. Neelakantan N, Naidoo N, Koh W-P et al. (2016) The Alternative Healthy Eating Index is associated with a lower risk of fatal and nonfatal acute myocardial infarction in a Chinese adult population. J Nutr 146, 13791386.

36. Piepoli MF, Hoes AW, Agewall S et al. (2016) 2016 European Guidelines on cardiovascular disease prevention in clinical practice. Eur Heart J 37, 2315-2381.

37. Han SL, Auer R, Cornuz J et al. (2016) Clinical nutrition in primary care: an evaluation of resident physicians' attitudes and self-perceived proficiency. Clin Nutr ESPEN 15, 69-74.

38. US Department of Health and Human Services \& US Department of Agriculture (2015) Dietary Guidelines for Americans 2015-2020, 8th edition. Washington, DC: US Goovernment Printing Office.

39. Macready AL, George TW, Chong MF et al. (2014) Flavonoid-rich fruit and vegetables improve microvascular reactivity and inflammatory status in men at risk of cardiovascular disease - FLAVURS: a randomized controlled trial. Am J Clin Nutr 99, 479-489.

40. Vitaglione P, Mennella I, Ferracane R et al. (2015) Wholegrain wheat consumption reduces inflammation in a randomized controlled trial on overweight and obese subjects with unhealthy dietary and lifestyle behaviors: role of polyphenols bound to cereal dietary fiber. Am J Clin Nutr 101, 251-261.

41. Freedman LS, Commins JM, Moler JE et al. (2014) Pooled results from 5 validation studies of dietary self-report instruments using recovery biomarkers for energy and protein intake. Am J Epidemiol 180, 172-188. 